\title{
Phosphoenolpyruvate Carboxykinase Deficiency
}

National Cancer Institute

\section{Source}

National Cancer Institute. Phosphoenolpyruvate Carboxykinase Deficiency. NCI

Thesaurus. Code C99015.

A very rare, autosomal recessive inherited disorder caused by deficiency of the enzyme phosphoenolpyruvate carboxykinase, which is involved in gluconeogenesis. It presents with hypoglycemia, failure to thrive, metabolic acidosis, muscle weakness, and hepatomegaly. 